> Lorsqu'ils quittent le testicule, les spermatozoïdes sont fonctionnellement immatures et disposent de moyens de défense intrinsèques limités. Ils deviendront mobiles et fécondants après leur descente et leur maturation progressive dans le tubule épididymaire. L'épididyme assure aussi la survie et la protection des gamètes mâles pendant cette phase de maturation posttesticulaire et pendant la période de stockage entre deux éjaculations. Parmi les agressions communes auxquelles les spermatozoïdes sont soumis, le stress oxydant occupe une place particulière et ambiguë. Les événements de la maturation épididymaire requièrent un certain niveau d'oxydation alors que le gamète mâle y est, par ailleurs, particulièrement sensible. Un équilibre très fin entre oxydation ménagée bénéfique et stress oxydant délétère est ainsi maintenu dans l'environnement épididymaire. Les enzymes antioxydantes de la famille des peroxydases à glutathion à activité thiol peroxydase jouent un rôle clé dans le contrôle de cet équilibre. <

Un couple sur six dans le monde consulte en raison de difficultés à concevoir. Quand on retrace les origines de ces infertilités, un tiers mettent en cause les deux partenaires, un tiers sont uniquement d'origine féminine et un tiers uniquement d'origine masculine. Dans ce dernier groupe, il apparaît que, dans 50 \% des cas, l'infertilité mâle est associée à un défaut de la spermatogenèse testiculaire. Pour l'autre moitié de ces infertilités mâles idiopathiques, la spermatogenèse est normale, mais c'est en aval du testicule que se situent les problèmes, essentiellement pendant la phase de maturation et de stockage épididymaire des spermatozoïdes. II est donc important d'étudier les phénomènes qui accompagnent cette maturation post-testiculaire des gamètes, et comment ces phénomènes peuvent être perturbés afin de comprendre et éventuellement de proposer des solutions thérapeutiques adaptées à ces patients infertiles.

\section{Protection post- testiculaire des gamètes mâles contre les dommages radicalaires}

\section{Le rôle de l'épididyme}

Anaïs Noblanc, Ayhan Kocer, Joël R. Drevet

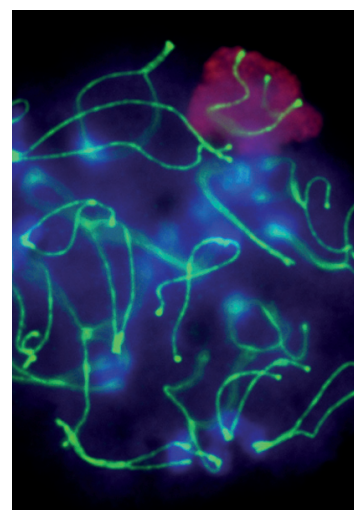

Laboratoire génétique, reproduction et développement (GReD), CNRS UMR 6293, Clermont Université, Inserm U1103, 24, avenue des Landais, 63177 Aubière Cedex, France. joel.drevet@univ-bpclermont.fr

\section{Fonctions de l'épididyme dans la maturation et la protection des spermatozoïdes}

L'épididyme est un organe accolé au testicule, formé d'un très long tubule, fortement contourné, qui met en communication le testicule en amont via le rete testis et les canaux efférents avec le canal déférent en aval. C'est un dérivé du mésonéphros embryonnaire apparu chez les amniotes (reptiles, oiseaux, mammifères) qui atteint son plein développement chez les mammifères. Chez ces derniers, l'épididyme est impliqué dans le transport et la maturation des spermatozoïdes ainsi que, dans sa partie terminale (queue ou cauda), dans le stockage des spermatozoïdes entre deux éjaculations [1-3] (Figure 1). La maturation post-testiculaire des spermatozoïdes recouvre un ensemble de processus complexes qui vont progressivement modifier structurellement et fonctionnellement les gamètes en transit et ainsi leur conférer leurs aptitudes fécondantes, c'est-à-dire l'expression de leur motilité et la capacité à reconnaître la zone pellucide de l'ovule et à fusionner avec ce dernier. L'épithélium composite pseudostratifié du tubule épididymaire assure ces rôles essentiellement grâce à ses multiples activités de sécrétion et de réabsorption qui changent localement et séquentiellement la composition du fluide épididymaire dans lequel baignent les gamètes (pour une revue récente voir [4]).

Une expérience simple qui illustre l'importance de ce tissu dans la fertilité mâle chez les mammifères consiste à tester les capacités fécondantes de gamètes prélevés dans différentes régions du tubule 


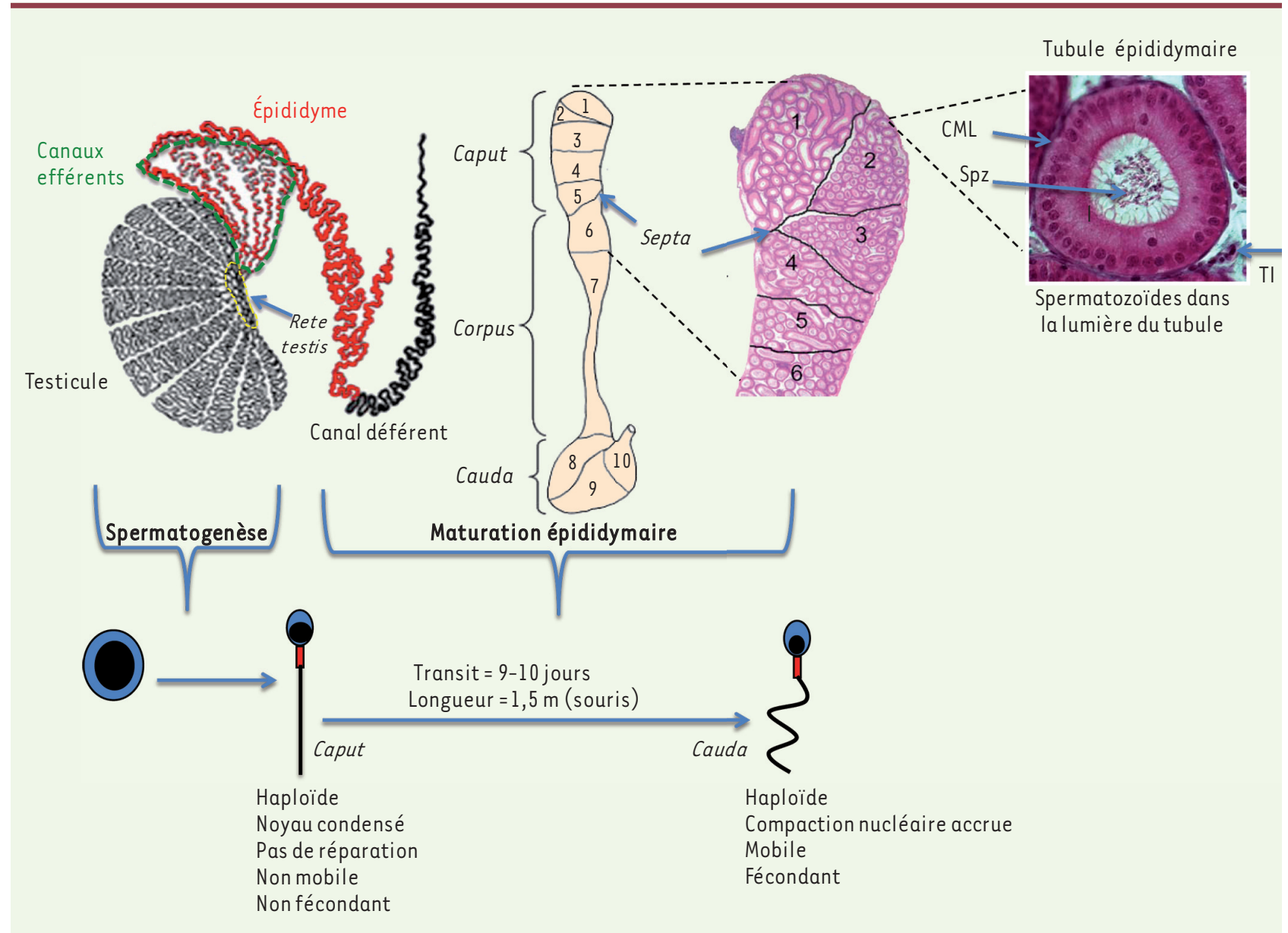

Figure 1. L'épididyme des mammifères. Les illustrations font référence au modèle murin. L'épididyme est le tubule rouge relié au hile du testicule via les canaux efférents et qui se poursuit ensuite par le canal déférent. L'épididyme est organisé en trois grandes régions anatomiques : la tête (ou caput), le corps (ou corpus) et la queue (ou cauda), elles-mêmes subdivisées en segments (numérotés de 1 à 10 chez la souris) séparés par des cloisons conjonctives (ou septa). Un seul et même tubule fortement pelotonné parcourt l'ensemble de l'organe. Le tubule est limité par un épithélium sécréteur pseudostratifié, lui-même entouré d’une assise de cellules musculaires lisses dont les contractions péristaltiques poussent les spermatozoïdes situés dans la lumière vers la partie terminale de l'organe, lieu de stockage des gamètes mâles entre deux éjaculations. À l'issue de la spermatogenèse testiculaire, les gamètes mâles haploïdes et cytodifférenciés sont des cellules quiescentes, incapables de se défendre et de mettre en jeu des processus de réparation. À leur entrée dans le tubule épididymaire les gamètes mâles sont en outre fonctionnellement immatures, incapables de faire fonctionner leur flagelle, de reconnaître un gamète femelle et de fusionner avec lui. À la fin de la maturation épididymaire, les spermatozoïdes stockés dans la queue de l'épididyme ont acquis leur pouvoir fécondant et seront mobiles dans un contexte permissif (l'environnement des voies génitales femelles). CML : cellules musculaires lisses, Spz : spermatozoïdes, TI : tissu interstitiel.

épididymaire: les gamètes prélevés à leur entrée dans le tubule épididymaire ne sont ni mobiles ni fécondants. Motilité et pouvoir fécondant des spermatozoïdes augmentent pour les spermatozoïdes prélevés dans les régions plus distales du tubule épididymaire. Associé à cette maturation fonctionnelle post-testiculaire des spermatozoïdes, l'épididyme assure aussi un rôle de « contrôle qualité » (voir par exemple [5]). Le nombre de spermatozoïdes présentant des morphologies aberrantes diminue entre la région proximale et la région distale du tubule épididymaire suggérant que des processus de tri existent au cours de la maturation épididymaire. Enfin, l'épididyme assure la survie et la protection de cette cellule particulière qu'est le spermatozoïde. Ces processus de survie et de protection doivent être efficaces puisque le trajet épididymaire des gamètes est de l'ordre de 10 à 12 jours chez les mammifères, excluant les temps de stockage qui sont liés à l'activité sexuelle du mâle. La protection des spermatozoïdes en transit dans le tubule et stockés dans la queue de l'épididyme se doit d'être particulièrement efficace, car le gamète mâle est une cellule fragile. Cette fragilité repose sur l'état quiescent de cette cellule qui, à l'issue de la spermatogenèse, est quasi silencieuse en termes de transcription et de traduction. 


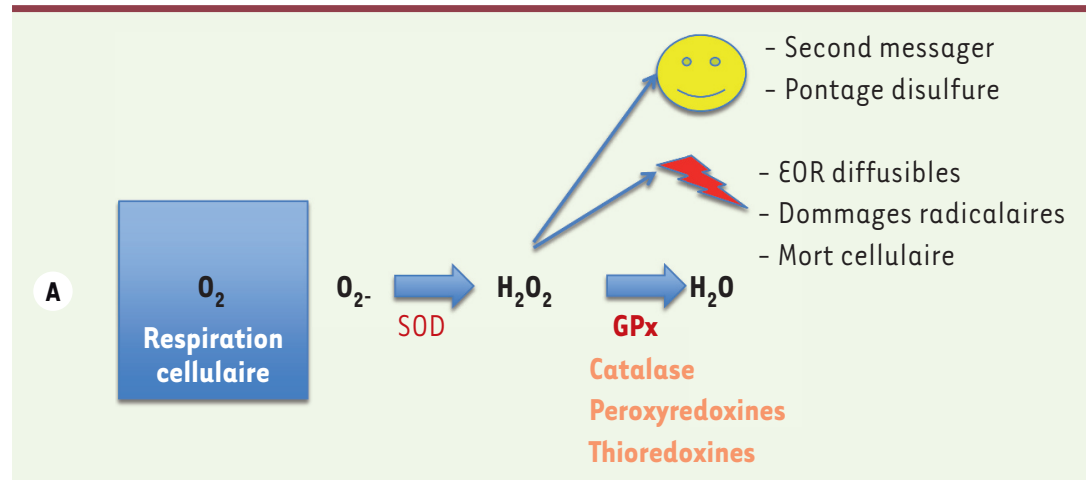

B GPx enzymes bifonctionnelles

$$
\begin{aligned}
& \text { GPx } \\
& \mathrm{H}_{2} \mathrm{O}_{2}+2 \mathrm{GSH} \rightarrow 2 \mathrm{H}_{2} \mathrm{O}+\mathrm{GSSG} \\
& \text { GPx } \\
& 2 x(\text { Protéine-SH })+\mathrm{H}_{2} \mathrm{O}_{2} \rightarrow \text { Protéine }-\mathrm{S}-\mathrm{S}-\text { Protéine }+2 \mathrm{H}_{2} \mathrm{O}
\end{aligned}
$$

Figure 2. Stress oxydant et enzymes antioxydantes. A. Les espèces oxygénées réactives et leur recyclage. La respiration cellulaire mitochondriale est le principal site de production d'espèces oxygénées réactives ( $(\varepsilon \cap)$ à partir de l'oxygène chez les eucaryotes aérobies. Le radical libre anion superoxyde $\left(\mathrm{O}_{2}^{-}\right)$est pris en charge par la superoxyde dismutase (SOD) qui le transforme en peroxyde d'hydrogène $\left(\mathrm{H}_{2} \mathrm{O}_{2}\right)$, une espèce activée de l'oxygène qui occupe une place carrefour dans cette voie de réduction univalente des $\varepsilon 0 \mathrm{R} . \mathrm{H}_{2} \mathrm{O}_{2}$ est nécessaire à la physiologie cellulaire en tant que second messager dans l'activation des voies de transduction de signaux, et en tant que donneur d'électrons dans la génération de ponts disulfures au niveau des groupements thiols libres portés par les résidus cystéine de nombreuses protéines. Dans cette deuxième fonction, $\mathrm{H}_{2} \mathrm{O}_{2}$ agit comme un intermédiaire clef dans la maturation structurale et fonctionnelle de nombreuses protéines cellulaires. Cependant, accumulé de façon trop importante en intracellulaire comme en extracellulaire, $\mathrm{H}_{2} \mathrm{O}_{2}$ est à l'origine de dommages radicalaires (stress oxydant) qui affectent tous les composés cellulaires (lipides membranaires, glucides, protéines et acides nucléiques) conduisant à terme à la mort cellulaire. Afin d'ajuster finement les concentrations intracellulaires et extracellulaires en $\mathrm{H}_{2} \mathrm{O}_{2}$, les cellules des eucaryotes aérobies utilisent une batterie d'antioxydants primaires non enzymatiques (glutathion, thioredoxine, vitamines) et enzymatiques (peroxydases à glutathion [GPx], catalase, peroxyredoxines, enzyme à activité thioredoxine-like). B. Les peroxydases à glutathion, des enzymes bifonctionnelles. Les GPx peuvent assurer deux types de réactions, qui toutes deux consomment et donc recyclent $\mathrm{H}_{2} \mathrm{O}_{2}$. De façon classique, les GPx jouent le rôle d'enzymes anti-oxydantes en recyclant $\mathrm{H}_{2} \mathrm{O}_{2}$ en présence de deux molécules de glutathion (GSH). Dans des conditions limitantes en GSH, les GPx en présence d' $\mathrm{H}_{2} \mathrm{O}_{2}$ peuvent être impliquées dans la formation de ponts disulfures inter- et intraprotéines portant des groupements thiols libres.

L'absence de transcription résulte de la compaction extrême de son lot chromosomique haploïde n'autorisant plus l'accès de l'ADN à la machinerie transcriptionnelle. Concernant la traduction, même s'il a été montré que les spermatozoïdes post-testiculaires pouvaient traduire de nouvelles protéines [6], le niveau de cette traduction est particulièrement faible en raison, d'une part, de l'absence de transcription et, d'autre part, de l'élimination de la très grande majorité du cytoplasme du spermatozoïde et des organites subcellulaires qu'il contient [38] $(\rightarrow)$, lesquels supportent la

$\rightarrow$ Voir $\mathrm{m} / \mathrm{s} \mathrm{n}^{\circ} 4$, avril 2012, page 343 traduction comme dans toute cellule. Ces deux caractéristiques structurales (compaction du noyau haploïde et élimination du cytoplasme à la spermiation) très particulières au spermatozoïde sont acquises pendant la dernière phase de la cytodifférenciation testiculaire des gamètes ou spermiogenèse $[7,8]$. Ainsi, les gamètes mâles quittant le testicule n'ont pas la possibilité de répondre de façon autonome à une quelconque agression, ni en déclenchant une réponse au stress reposant sur l'engagement de gènes de défense, ni en mobilisant leurs maigres ressources cytoplasmiques en protéines de défense. Parmi les agressions auxquelles sont soumises les cellules, l'attaque radicalaire ou stress oxydant [9] constitue la plus commune et toutes les cellules d'un organisme aérobie y sont exposées. Plus que toute autre cellule, le spermatozoïde est susceptible de subir des dommages radicalaires. La sensibilité du gamète mâle vis-à-vis de l'attaque radicalaire est en effet accrue par la composition lipidique particulière de sa membrane plasmique, riche en acides gras polyinsaturés, cibles de choix des espèces oxygénées réactives ( $(\varepsilon 0 R)$.

\section{Le stress oxydant : un danger majeur pour les spermatozoïdes post-testiculaires}

\section{Dualité d'action de $\mathrm{H}_{2} \mathrm{O}_{2}$}

Toutes les cellules des organismes aérobies consomment de l'oxygène pour produire de l'énergie qui participe aux phosphorylations oxydatives et au métabolisme cellulaire. Ce faisant, elles génèrent des dérivés actifs de l'oxygène (DAO) ou EOR. Il est nécessaire de contrôler ces dérivés en raison de leur réactivité élevée et des réactions en chaîne qu'ils produisent lorsqu'ils attaquent des composés organiques, que ce soit à l'intérieur ou à l'extérieur des cellules (Figure 2A) [9]. Pour contrecarrer les effets nocifs de ces $\varepsilon 0 R$, les cellules utilisent une batterie d'antioxydants primaires, enzymatiques et non enzymatiques, cytosoliques, membranaires et sécrétés. L'anion superoxyde $\left(\mathrm{O}_{2}^{-}\right)$, premier dérivé actif de l'oxygène, réactif sur son site de production mais peu mobile, est transformé en peroxyde d'hydrogène $\left(\mathrm{H}_{2} \mathrm{O}_{2}\right)$ par la superoxyde dismutase (SOD). Cette espèce activée de l'oxygène est une espèce 


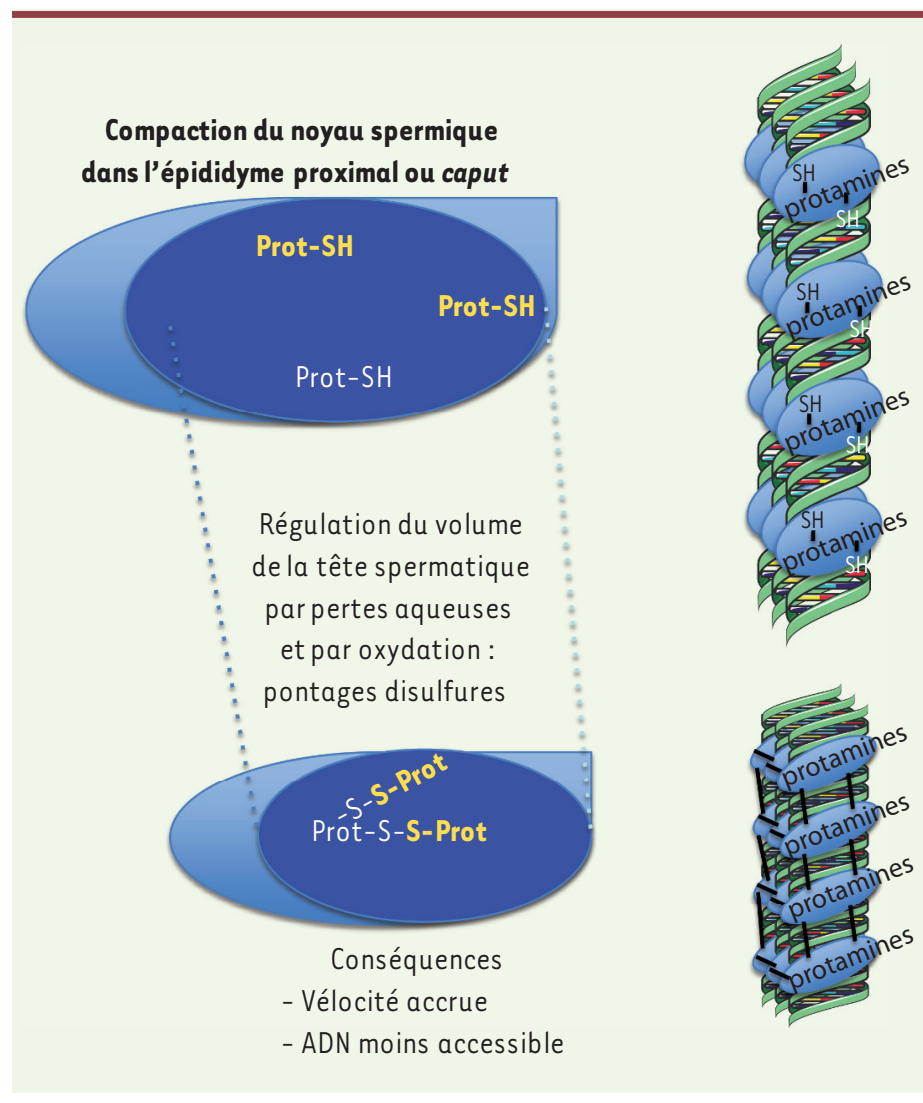

carrefour importante, potentiellement nocive mais aussi nécessaire dans plusieurs aspects du métabolisme cellulaire. À cause de son pouvoir de diffusion important, cet $\varepsilon 0 R$ est plus dangereux que l'anion superoxyde. En présence d'oxygène et de fer, selon les réactions biochimiques classiques de Fenton et d'Haber-Weiss, $\mathrm{H}_{2} \mathrm{O}_{2}$ pourra donner naissance à de nouveaux radicaux libres très agressifs (comme le radical hydroxyle) contre lequel les cellules peuvent peu ou pas se défendre, car elles sont dépourvues d'outils enzymatiques appropriés. Ces radicaux libres finiront par agresser tous les compartiments cellulaires, à commencer par les lipides dans les membranes cellulaires, perturbant leur fonction d'échange. Les dommages radicalaires s'étendront ensuite à tous les autres composés cellulaires y compris à l'ADN dans le compartiment nucléaire. Une accumulation trop importante de ces $\varepsilon 0 \mathrm{R}$ peut alors conduire à la mort cellulaire [10]. À côté de ce rôle délétère, $\mathrm{H}_{2} \mathrm{O}_{2}$ a cependant des rôles bénéfiques indispensables à la physiologie cellulaire. Tout le monde connaît le rôle antibactérien du peroxyde d'hydrogène et son implication dans la réponse immune et dans l'inflammation. Moins connu, mais cependant très important, est le rôle de $\mathrm{H}_{2} \mathrm{O}_{2}$ dans la maturation des protéines via la création, spontanée ou assistée par des enzymes, de ponts disulfures sur les résidus thiols portés par les acides aminés cystéines. Tout aussi important est le rôle de $\mathrm{H}_{2} \mathrm{O}_{2}$ comme second messager inter- et intracellulaire, acteur dans la mise en $œ u v r e$ et la modulation de voies de transduction de signaux. Ceci explique que les concentrations intracellulaires et extracellulaires en $\mathrm{H}_{2} \mathrm{O}_{2}$ doivent être finement régulées. À cet effet, les cellules des eucaryotes aérobies utilisent un ensemble d'enzymes antioxydantes telles que la catalase,
Figure 3. Condensation post-testiculaire du noyau spermatique. La compaction du noyau spermatique, qui débute lors de la spermiogenèse testiculaire par le remplacement des histones nucléosomales par les protamines, est poursuivie dans la tête de l'épididyme par l'oxydation des groupements thiols libres portés par les nombreux résidus cystéine contenus dans les protamines. Des mouvements d'eau et d'ions provoquent aussi dans ce compartiment proximal de l'épididyme des pertes aqueuses qui contribuent à la diminution de la taille de la tête spermatique. Ces processus de condensation/compaction accrue permettent au spermatozoïde d'acquérir une vélocité optimale et confèrent au noyau spermatique, donc au patrimoine génétique paternel, une protection plus importante contre d'éventuels effets mutagènes.

les peroxydases à glutathion (GPx), les peroxyredoxines (PRDX) et les thiol peroxydases (TXN-like) de façon à recycler $\mathrm{H}_{2} \mathrm{O}_{2}$ (Figure 2A).

\section{L'environnement épididymaire pro-oxydant}

Cette dualité d'action de $\mathrm{H}_{2} \mathrm{O}_{2}$ sur les cellules, à la fois délétère et bénéfique, est mise en exergue lors de la maturation post-testiculaire des spermatozoïdes au sein de l'épididyme [11]. En effet, comme nous l'avons vu ci-dessus, les spermatozoïdes sont très sensibles aux attaques oxydantes et utilisent $\mathrm{H}_{2} \mathrm{O}_{2}$ et/ou des dérivés lipidiques hydroperoxydés ( $\mathrm{LOOH}$ ) pour parfaire leur maturation. Plusieurs protéines spermatiques membranaires mais aussi internes au gamète sont soumises à une intense activité de pontage disulfure pendant la descente épididymaire [12-19]. L'évolution du rapport thiols libres/disulfides des protéines spermatiques en tête $(10 / 1)$ et en queue d'épididyme (1/10) illustre ce phénomène. Les protamines nucléaires, qui ont remplacé les histones nucléaires pendant la spermiogenèse, sont parmi les protéines spermatiques soumises à cette activité d'oxydation. Le pontage inter- et intraprotamines qui en résulte au cours de la descente dans l'épididyme participe à la poursuite de la diminution de la taille de la tête spermatique et au verrouillage du noyau spermatique dans un état condensé (Figures 1, $2 B$ et 3 ). Cette compaction accrue du noyau spermatique, et donc de la tête du spermatozoïde, au cours de la descente épididymaire est un événement important de la maturation post-testiculaire des gamètes contribuant à l'acquisition d'une vélocité optimale et à la protection du lot chromosomique paternel, deux critères essentiels du succès reproductif. Ces événements de pontage disulfure des protéines spermatiques 


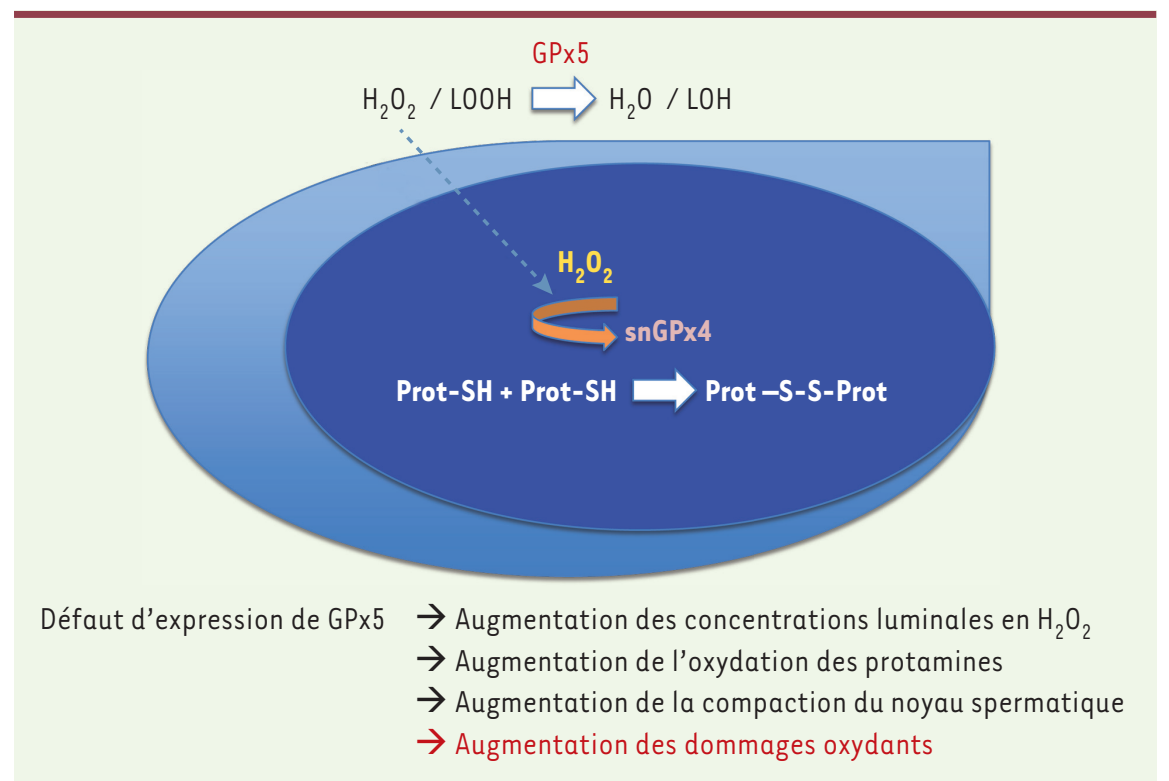

Défaut d'expression de snGPx4 $\rightarrow$ Décondensation du noyau spermatique

Figure 4. Rôles des GPX dans l'équilibre du stress oxydant dans le compartiment épididymaire. La glutathion peroxydase 5 (GPx5) sécrétée par l'épithélium de l'épididyme proximal contrôle le niveau luminal en $\mathrm{H}_{2} \mathrm{O}_{2}$. Indirectement, elle définit par cette action le niveau optimal de pontage disulfure pouvant être réalisé sur les résidus thiols des protamines par le variant spermatique nucléaire de la GPx4 (snGPx4). L'analyse des modèles murins mutants invalidés pour ces activités GPx a montré que des défauts d'activité GPx5 luminale provoquent transitoirement dans la tête de l'épididyme une compaction plus importante du noyau spermatique liée à une activité d'oxydation par snGPx4 accrue en raison de la plus grande disponibilité en $\mathrm{H}_{2} \mathrm{O}_{2}$. Cependant, in fine, l'exposition luminale des gamètes à des quantités d' $\mathrm{H}_{2} \mathrm{O}_{2}$ plus importantes est source de dommages radicalaires (peroxydation des lipides membranaires, atteintes oxydantes du noyau spermatique) pouvant affecter I'issue de la fécondation [21]. En parallèle, un défaut d'expression de la GPx4 du noyau spermatique entraîne des défauts de condensation du noyau spermatique dans la tête de l'épididyme [20].

incluant les protamines nucléaires requièrent un environnement épididymaire pro-oxydant (riche en $\mathrm{H}_{2} \mathrm{O}_{2}$ et/ou en $\mathrm{LOOH}$ ) qu'il est nécessaire de contrôler de façon très fine, étant donné le risque de dommages oxydatifs. Des enzymes antioxydantes épididymaires assurent cet équilibre [11].

\section{Le double rôle des enzymes antioxydantes de la famille des peroxydases à glutathion, GPx}

En relation avec cette nécessité de préserver les fragiles gamètes mâles des dommages oxydants, nous avons démontré ces dernières années que l'épididyme des mammifères possède une couverture antioxydante importante. Cela se manifeste par l'expression de multiples formes d'antioxydants enzymatiques primaires dont certains sont même d'expression majoritaire ou quasi restreinte à cet organe [20]. C'est le cas de la glutathion peroxydase 5 (GPx5), une enzyme abondamment sécrétée par l'épithélium de la tête de l'épididyme qui accompagne les gamètes mâles tout le long de leur descente dans le tubule épididymaire. Plusieurs autres GPx cytosoliques (GPxl, GPx3 et cGPx4) sont par ailleurs exprimées par l'épithélium épididymaire [20]. Le gamète mâle lui-même porte des GPx qui lui sont spécifiques, suite à l'expression de variants testiculaires de la GPx4 au cours de la spermatogenèse. C'est le cas de la snGPx4, un variant d'expression de la GPx4 localisé dans le noyau spermatique et de la mGPx4, un autre variant d'expression de la GPx4 localisé au niveau des mitochondries de la pièce intermédiaire. À côté de cette expression importante de multiples GPx, l'épididyme et les gamètes mâles présentent par ailleurs une couverture en peroxyredoxines (PRDX) et en diverses thiol-peroxydases [21].

Les GPx sont des enzymes bifonctionnelles pouvant fonctionner comme des recycleurs d'EOR (en particulier I' $\mathrm{H}_{2} \mathrm{O}_{2}$, mais aussi les $\mathrm{LOOH}$ ) ou comme des intermédiaires dans la formation de ponts disulfures (Figure 2B). Cette dualité fonctionnelle est particulièrement bien illustrée au cours de la maturation épididymaire des spermatozoïdes. Ainsi, Conrad et al. [22] ont démontré que la GPx4 du noyau spermatique (snGPx4) était associée aux protamines et impliquée dans la formation de ponts disulfures intra- et interprotamines entre les groupements thiols portés par les nombreux résidus cystéines présents dans ces protéines nucléaires. C'est lors du passage dans la tête de l'épididyme que l'essentiel de cette activité de pontage disulfure est assurée par la snGPx4, dans un environnement qui doit fournir le $\mathrm{H}_{2} \mathrm{O}_{2}$ ou les $\mathrm{LOOH}$ nécessaires. Cette activité de pontage disulfure du noyau spermatique dans l'épididyme proximal contribue à diminuer encore la taille du noyau spermatique et à verrouiller le noyau spermatique dans un état condensé (Figure 3). Ces aspects sont particulièrement importants puisque, d'une part, ils définissent la taille de la tête spermatique qui ellemême conditionne la vélocité progressive rectiligne du spermatozoïde et, d'autre part, ils augmentent la protection du matériel nucléaire paternel contre les dommages à I'ADN de type agents intercalants ou molécules mutagènes. Ces deux caractéristiques, 
vélocité optimale et matériel génétique intègre, sont les garants du succès reproductif.

L'ambiguïté réside dans le fait que pour assurer ces pontages disulfures, les gamètes mâles particulièrement sensibles aux dommages radicalaires doivent évoluer dans un environnement plutôt pro-oxydant. Il est donc nécessaire de contrôler de façon efficace le niveau d'£OR dans le tubule épididymaire. C'est ici que la GPx5 sécrétée par l'épithélium de l'épididyme proximal intervient. La génération récente d'un modèle murin n'exprimant plus la GPx5, celui des souris $g p \times 5^{-/-}$[23], a permis de révéler que cette enzyme fonctionne dans la lumière du tubule épididymaire comme un nettoyeur d'દ0R, protégeant les spermatozoïdes contre les attaques radicalaires. Dans la tête de l'épididyme, le rôle de la GPx5 est double. D'une part, elle protège les gamètes de l'oxydation et, d'autre part, elle fixe le niveau de pontage disulfure en contrôlant la concentration luminale en $\mathrm{H}_{2} \mathrm{O}_{2}$ et/ou en $\mathrm{LOOH}$. Le rôle de contrôleur indirect de la compaction du noyau spermatique en tête d'épididyme a été révélé par le fait que le noyau des spermatozoïdes de la tête d'épididyme de souris $g p \times 5^{-/-}$est sensiblement plus compacté que celui des spermatozoïdes des souris sauvages. Cette compaction accrue pourrait être due à la plus grande disponibilité en $\mathrm{H}_{2} \mathrm{O}_{2}$ et/ou $\mathrm{LOOH}$ favorisant ainsi l'activité snGPx4 en raison de l'absence de recyclage de ces EOR par GPx5 [11, 23] (Figure 4). En queue d'épididyme, la GPx5 assure essentiellement un rôle de protection des gamètes stockés contre les dommages radicalaires. En effet, les spermatozoïdes des animaux dépourvus de GPx5 présentent une peroxydation lipidique membranaire accrue et subissent des dommages oxydants à l'ADN révélés par la présence de bases oxydées et d'un noyau susceptible à la fragmentation. Dans ce fond génétique $g p x^{-/-}$, ces atteintes de l'intégrité du noyau paternel ont été corrélées (essentiellement chez les mâles âgés) à une augmentation du nombre de fœtus avortés et du nombre d'accidents de développements conduisant à des fœtus malformés, ainsi qu'à une augmentation de la mortalité périnatale [23].

\section{Conclusions}

Ces observations réalisées à l'aide de ces modèles murins transgéniques ont permis d'améliorer notre perception des événements de la maturation post-testiculaire des spermatozoïdes. Longtemps considéré comme un simple tuyau, l'épididyme apparaît clairement maintenant comme un tissu essentiel à la fertilité mâle. Les phénomènes mis en exergue ici trouvent leur écho en clinique, comme en témoigne une littérature pléthorique qui atteste qu'une fraction importante des infertilités mâles est corrélée à des dommages oxydants des spermatozoïdes affectant la mobilité progressive, perturbant la capacité à réaliser la réaction acrosomique et conduisant à un niveau élevé de fragmentation nucléaire [24-37]. Cet équilibre précaire entre oxydation ménagée bénéfique et dommages oxydatifs au gamète mâle explique sans doute le fait que les dommages radicalaires au spermatozoïde soient une cause si fréquente de l'infertilité mâle, qu'elle soit d'origine génétique, métabolique, secondaire aux expositions environnementales, ou tout simplement liée à l'âge. Oxydation et fragmentation du noyau spermatique sont de même corrélées au succès reproductif en procréation médicalement assistée (PMA). En outre, les technologies de la PMA peuvent elles-mêmes être à l'origine d'atteintes oxydatives du gamète mâle (par exemple l'utilisation de sperme congelé qui subit une explosion oxydative lors de son réchauffement). Si une mobilité spermatique diminuée et/ou une capacité à déclencher la réaction acrosomique perturbée vont diminuer les taux de fécondation, les dommages radicalaires au noyau paternel sont autrement plus dangereux. L'ovule est sensé réparer les dommages à l'ADN paternel et ses capacités de réparation sont grandes. Cependant, dans un contexte de réparation déficiente, comme cela peut-être le cas dans un ovule non optimal (femme âgée, PMA avec une «superovulation » provoquée par l'administration d'hormones, PMA via l'injection intracytoplasmique de spermatozoïdes [ICSI]), certains dommages à l'ADN courent le risque d'être transmis à la descendance et d'être à l'origine de pathologies. $\diamond$

\section{SUMMARY}

Post-testicular protection of male gametes from oxidative damage. The role of the epididymis Spermatozoa leave the testis in an immature functional state and are devoid of self defense mechanisms. They will become motile and ready to fertilize only after their descent and their progressive maturation within the epididymal tubule. The epididymis also ensures the survival and the protection of male gametes while they go through the epididymis and during their storage in between two ejaculations. Amongst common stresses that concern spermatozoa, oxidative stress occupies a peculiar and dual position. While the events of epididymal sperm maturation necessitate a given level of oxidation, spermatozoa are particularly sensitive to oxidative damage. A fine balance between beneficial oxidation versus detrimental oxidative damage has to be maintained in the epididymal environment. Antioxidant enzymes of the glutathione peroxidase family play a key role in controling such a situation in the epididymis. $\diamond$

\section{LIENS D'INTÉRÊT}

Les auteurs déclarent n'avoir aucun lien d'intérêt concernant les données publiées dans cet article.

\section{RÉFÉRENCES}

1. Orgebin-Crist MC, Danzo BJ, Davies J. Endocrine control of the development and maintenance of sperm fertilizing ability in the epididymis. In : Greep R, Hamilton DW, eds. Handbook of physiology-endocrinology V. Baltimore : Williams and Wilkins, $1975: 319-38$.

2. Bedford JM. Maturation, transport and fate of spermatozoa in the epididymis. In : Greep R, Hamilton DW, eds. Handbook of physiologyendocrinology V. Baltimore: Williams and Wilkins, 1975 : 302-17. 


\section{RÉFÉRENCES}

3. Hinton BT, Palladino MA, Rudolph D, Labus JC. The epididymis as protector of maturing spermatozoa. Reprod Fertil Dev $1995 ; 7: 731-43$.

4. Cornwall GA. New insights into epididymal biology and function. Hum Reprod Update $2009 ; 15$ : 213-27.

5. Cornwall GA, von Horsten HH, Swartz D, et al. Extracellular quality control in the epididymis. Asian J Androl $2007 ; 9$ : $500-7$

6. Gur Y, Breibart H. Protein synthesis in sperm : dialog between mitochondria and cytoplasm. Mol Cell Endocrinol $2008 ; 282: 45-53$.

7. Ward WS, Coffey DS. DNA packaging and organization in mammalian spermatozoa : comparison with somatic cells. Biol Reprod $1991 ; 44: 569-74$

8. Miller D, Brinkworth M, lles D. Paternal DNA packaging in spermatozoa : more than the sum of its parts? DNA, histones, protamines and epigenetics. Reproduction $2010 ; 139$ : 287-301.

9. Migdal C, Serres M. Espèces oxygénées réactives et stress oxydant. Med Sci (Paris) 2011 ; 27 : 405-12.

10. Halliwell B, Gutteridge JM. Lipid peroxidation, oxygen radicals, cell damage, and antioxidant therapy. Lancet $1984 ; 1: 1396-7$.

11. Noblanc A, Kocer A, Chabory $\varepsilon$, et al. Glutathione peroxidases at work on epididymal spermatozoa : an example of the dual effect of reactive oxygen species on mammalian male fertilizing ability. J Androl $2011 ; 32: 641-50$.

12. Calvin HI, Bedford JM. Formation of disulphide bonds in the nucleus and accessory structures of mammalian spermatozoa during maturation in the epididymis. J Reprod Fertil $1971 ; 13: 65-75$.

13. Bedford JM, Calvin HI. Changes in -S-S- linked structures of the sperm tail during epididymal maturation, with comparative observations in sub-mammalian species. J Exp Zool 1974 ; 187 : 137-56.

14. Shalgi R, Seligman J, Kosover NS. Dynamics of the thiol status of rat spermatozoa during maturation: analysis with the fluorescent labeling agent monobromobimane. Biol Reprod 1989; $40: 1037-45$

15. Seligman J, Kosower NS, Weissenberg R, Shalgi R. Thiol-disulfide status of human sperm proteins. J Reprod Fertil $1994 ; 101: 435-54$.

16. Balhorn R, Corzett M, Mazrimas J, Watkins B. Identification of bull protamine disulfides. Biochemistry $2001 ; 30: 175-81$.

17. Yeung CH, Barfield JP, Cooper TG. Physiological volume regulation by spermatozoa. Mol Cell Endocrinol $2006 ; 250$ : 98-105.

18. Huang HF, Nieschlag $\varepsilon$. Alteration of free sulphydryl content of rat sperm heads by suppression of intratesticular testosterone. J Reprod Fertil $1984 ; 70: 31-8$.

19. Golan R, Cooper TG, Oschry Y, et al. Changes in chromatin condensation of human spermatozoa during epididymal transit as determined by flow cytometry. Hum Reprod 1996 ; 11 : 1457-62.

20. Drevet JR. The antioxidant glutathione peroxidase family and spermatozoa: a complex story. Mol Cell Endocrinol $2006 ; 250: 70-9$.

21. O'Flaherty C, de Lamirande $\varepsilon$, Gagnon C. Positive role of reactive oxygen species in mammalian sperm capacitation: triggering and modulation of phosphorylation events. Free Radic Biol Med $2006 ; 41: 528-40$.

22. Conrad M, Moreno SG, Sinowatz F, et al. The nuclear form of phospholipids hydroperoxide glutathione is a protein thiol peroxidase contributing to sperm chromatin stability. Mol Cell Biol $2005 ; 25: 7637-44$.
23. Chabory $\varepsilon$, Damon $C$, Lenoir $A$, et al. Epididymis seleno-independent glutathione peroxidase 5 (GPx5) contributes to the maintenance of sperm DNA integrity. J Clin Invest $2009 ; 119$ : 2074-85.

24. Kodama H, Yamaguchi R, Fukuda J, et al. Increased oxidative deoxyribonucleic acid damage in the spermatozoa of infertile male patients. Fertil Steril $1997 ; 68: 519-24$

25. Spano M, Bonde JP, Hjollund HI, et al. Sperm chromatin damage impairs human fertility. The Danish first pregnancy planner study team. Fertil Steril $2000 ; 73: 43-50$.

26. Zini A, Bielecki R, Phang D, Zenzes MT. Correlations between two markers of sperm DNA integrity, DNA denaturation and DNA fragmentation, in fertile and infertile men. Fertil Steril $2001 ; 75: 674-77$.

27. Tesarik J, Greco E, Mendoza C. Late, but not early, paternal effect on human embryo development is related to sperm DNA fragmentation. Hum Reprod $2004 ; 19: 611-5$.

28. Lewis SE, Aitken RJ. DNA damage to spermatozoa has impacts on fertilization and pregnancy. Cell Tissue Res $2005 ; 4$ : 657-66.

29. 0'Brien J, Zini A. Sperm DNA integrity and male infertility. Urology $2005 ; 65$ $16-22$

30. Zini A, Libman J. Sperm DNA damage: importance in the era of assisted reproduction. Curr Opin Urol $2006 ; 16: 428-34$.

31. Zini A, Libman J. Sperm DNA damage: clinical significance in the era of assisted reproduction. CMAJ $2006 ; 175: 495-500$.

32. Tesarik J, Mendoza-Tesarik R, Mendoza C. Sperm nuclear DNA damage: update on the mechanism, diagnosis and treatment. Reprod Biomed Online $2006 ; 12: 715-21$

33. Cocuzza M, Sikka SC, Athayde KS, Agarwal A. Clinical relevance of oxidative stress and sperm chromatin damage in male infertility: an evidence based analysis. Int Braz J Urol 2007 ; $33: 603-21$.

34. Marchesi DE, Feng HL. Sperm DNA integrity from sperm to egg. J Androl $2007 ; 28: 481-9$.

35. Aitken RJ, De Luliis GN, McLachlan RI. Biological and clinical significance of DNA damage in the male germ line. Int J Androl 2008 ; 32 : 46-56.

36. Aoki VW, Moskovtsev SI, Willis J, et al. DNA integrity is compromised in protamine-deficient human sperm. J Androl $2005 ; 26$ : 741-8.

37. Sakkas D, Alvarez JG. Sperm DNA fragmentation : mechanisms of origin, impact on reproductive outcome, and analysis. Fertil Steril $2010 ; 93: 1027-$ 36.

38. Al Rawi S, Galy V. L'allophagie, ou comment l'embryon élimine les mitochondries et autres organites paternels. Med Sci (Paris) 2012 ; 28 : 343-7.

\section{TIRÉS À PART}

J.R. Drevet

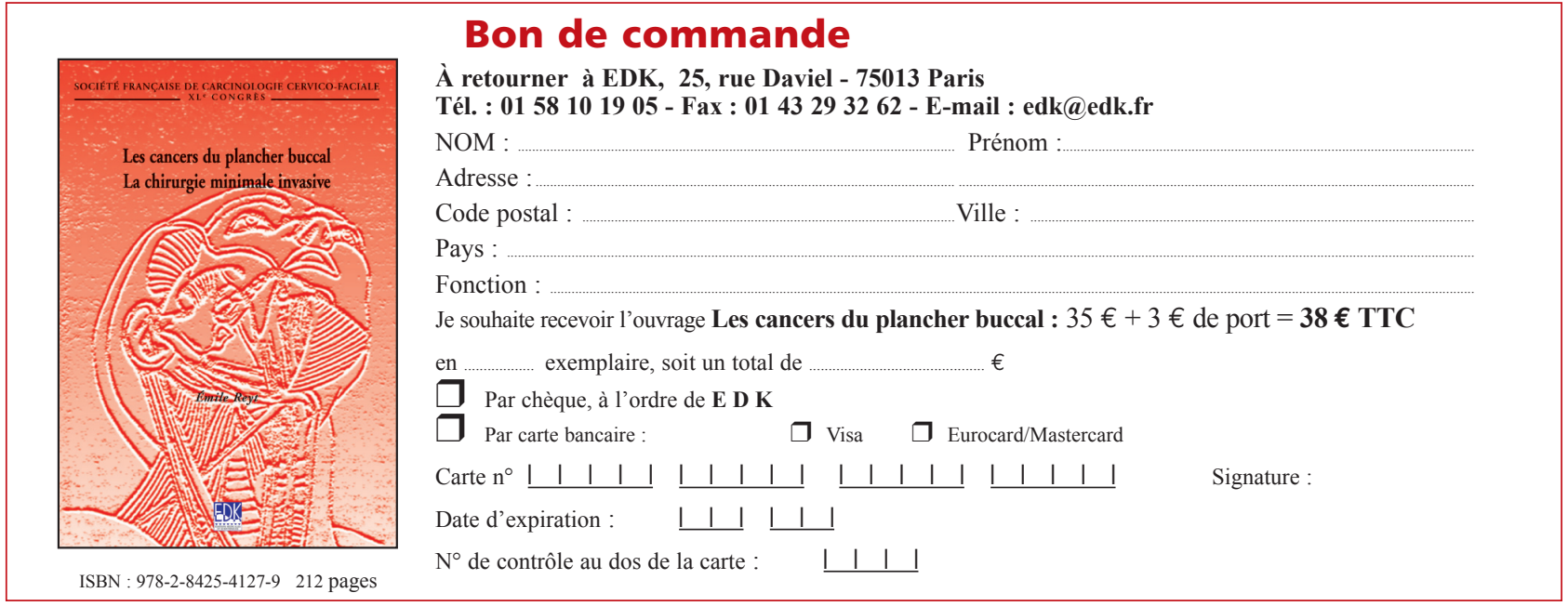

